\title{
UNBOUNDED TOEPLITZ OPERATORS IN THE SEGAL-BARGMANN SPACE, III
}

\author{
JAN JANAS
}

\section{Introduction}

The paper is a continuation of our previous study of unbounded Toeplitz operators in the Segal-Bargmann space; [6], [7]. This work is divided into separate sections, devoted to different topics described below.

In Section 2 some necessary preliminaries are given. Section 3 is devoted to the problem of computing the adjoint of Toeplitz operators. A class of entire symbols $\varphi$ is found for which $T_{\varphi}^{*}$ has been computed.

Section 4 deals with Toeplitz operators as generators of contraction semigroups. In turn Section 5 describes Fischer pairs of polynomials (the notion introduced by H. Shapiro in [11]), from the point of view of perturbation theory. The last Section 6 ends up with remarks and open problems. We thank anonymous referee for his helpful and careful remarks.

\section{Preliminaries}

In what follows we shall use notation introduced in the previous paper [7]. In particular, the standard multiindex notation will be used.

For a densely defined linear operator $T$ in a Hilbert space we denote by $T^{*}, \bar{T}, R(T), W(T)$, and $\sigma(T)$ the adjoint operator of $T$, the closure of $T$, the range of $T$, the numerical range of $T$ and the spectrum of $T$, respectively. A linear subspace $D$ contained in the domain $D(T)$ of $T$ is called a core of $T$ if $\left(T_{\mid D}\right)^{-}=T$. In what follows $D^{k}=D_{1}^{k_{1}} \cdots D_{n}^{k_{n}}$, where $D_{j}=\frac{\partial}{\partial z_{j}}, k=$ $\left(k_{1}, \cdots, k_{n}\right) \in \mathrm{N}^{n}$.

For a polynomial $p=\sum_{k} a_{k} z^{k}$ the operator $p^{*}(D)$ is given by $\sum_{k} \bar{a}_{k} D^{k}$.

For the reader's convenience we also briefly recall definitions concerning Toeplitz operators in the Segal-Bargmann space $B$. This space consists of all entire functions in $C^{n}$ which are square integrable with respect to the Gaussian measure $d \mu=\pi^{-n} \exp \left(-\|z\|^{2}\right) d V$, where $d V$ is the Lebesgue measure on

Received July 23, 1996; in revised form March 27, 1998. 
$\mathrm{C}^{n}$. Given a Borel function $\varphi: \mathrm{C}^{n} \rightarrow \mathrm{C}$ we denote by $M_{\varphi}$ the operator of multiplication by $\varphi$ in $L^{2}(\mu)$, where

$$
D\left(M_{\varphi}\right)=\left\{f \in L^{2}(\mu), \varphi f \in L^{2}(\mu)\right\} .
$$

By Toeplitz (resp. Hankel) operator with symbol $\varphi$ we mean the operator $T_{\varphi}$ (resp. $H_{\varphi}$ ) defined in $B$ by:

$$
D\left(T_{\varphi}\right)=D\left(M_{\varphi}\right) \cap B, \quad T_{\varphi} f=P M_{\varphi} f, \quad f \in D\left(T_{\varphi}\right),
$$

(resp. $H_{\varphi} f=(I-P) M_{\varphi} f$ ), where $P$ is the orthogonal projection of $L^{2}(\mu)$ onto $B$ and $I$ is the identity operator.

In turn, the domain $D\left(\Pi_{\varphi}\right)$ of the related operator $\Pi_{\varphi}$, already defined in [6], is the set of functions $f \in B$ such that the integral $\int \varphi(a) f(a) e^{<z, a>} d \mu(a):=\Pi_{\varphi} f(z)$ exists for each $z \in \mathrm{C}^{n}$ and $\Pi_{\varphi} f \in B$.

The function $e_{a}(z):=\exp \langle z, a\rangle$ is the reproducing kernel of $B$ and the sequence $f_{k}(z)=z^{k} / \sqrt{k !}$ is the orthonormal basis in $B$. Let $\mathscr{P}$ be the set of all polynomials in $\mathrm{C}^{n}$ and let $E$ be equal to the linear span of $\left\{e_{w}, w \in \mathrm{C}^{n}\right\}$.

\section{Computing $T_{\varphi}^{*}$}

The problem of computing the adjoint $T_{\varphi}^{*}$ of $T_{\varphi}$ has already been studied in [6], [7], [10]. Even for an entire symbol $\psi$ the conjecture $T_{\psi}^{*}=\Pi_{\bar{\psi}}$ is still open.

Below we present a positive answer to the conjecture for a class of entire symbols $\varphi$. This class turns out to be sufficiently large e.g. contains exponential polynomials [10], [7]. But it is far from being equal to the whole set of entire symbols satisfying the conjecture.

THEOREM 3.1. Let $\varphi$ be an entire function satisfying the following conditions: $E \subset D\left(T_{\varphi}\right)$

$$
D\left(T_{\varphi}\right) \subset D\left(T_{\varphi^{(s)}}\right), \quad s \in \mathrm{N}^{n}
$$

$(* *) \quad \sum_{s} \frac{\left|\varphi^{(s)}(w)\right|\left\|\varphi^{(s)} e_{w}\right\|}{s !}$ belongs to $L^{2}(\mu)$ as a function of $w$.

Then $T_{\varphi}^{*}=\Pi_{\bar{\varphi}}$.

Proof. First note that the series $\sum_{s} \overline{\varphi^{(s)}(w)} \varphi^{(s)}(\cdot) e_{w}(\cdot) / s !$ is convergent in $B$, for every $w \in \mathrm{C}^{n}$. We claim that

$$
\Pi_{\bar{\varphi}}\left(\varphi e_{w}\right)(\cdot)=\sum_{s} \overline{\varphi^{(s)}(w)} \varphi^{(s)}(\cdot) e_{w}(\cdot) / s !
$$


Fix $a \in C^{n}$. Since $\left(T_{e_{a \mid \mathscr{P}}}\right)^{-}=T_{e_{a}}$ there exists a sequence $p_{k} \in \mathscr{P}$ such that $p_{k} e_{a} \rightarrow \varphi e_{a}$ in $B$ as $k \rightarrow \infty$. Take any $\varepsilon>0$ and choose $n_{0}=n_{0}(\varepsilon, a, w)$ s.t.

$$
\sum_{|s|>n_{0}}\left|\varphi^{(s)}(w) \varphi^{(s)}(a) e_{w}(a)\right| / s !<\varepsilon / 2
$$

Note that

$$
\begin{aligned}
\mid \varphi^{(s)}(a) & -p_{k}^{(s)}(a)|=|\left(D^{s}\left(\varphi-p_{k}\right), e_{a}\right) \mid \\
& =\left|\left(T_{e_{a}}^{*}\left(\varphi-p_{k}\right), f_{s}\right)\right| \sqrt{s !}
\end{aligned}
$$

Denote $N_{k}=\operatorname{deg} p_{k}$. We may assume (by increasing $k$ ) that $N_{k} \geq n_{0}$. Choose $k_{0}$ so large that

$$
\left|e_{w}(a)\right|\left(\sum_{s}\left|\varphi^{(s)}(w)\right|^{2} / s !\right)^{\frac{1}{2}}\left\|\left(p_{k}-\varphi\right) e_{a}\right\|<\varepsilon / 2
$$

for all $k \geq k_{0}$

Then we have

$$
\begin{gathered}
A_{k}:=\left|\sum_{s} \overline{\varphi^{(s)}(w)}\left[\varphi^{(s)}(a)-p_{k}^{(s)}(a)\right] e_{w}(a) / s !\right| \\
\leq \sum_{|s| \leq N_{k}}\left|\varphi^{(s)}(w)\left[\varphi^{(s)}(a)-p_{k}^{(s)}(a)\right] e_{w}(a) / s !\right| \\
\quad+\sum_{|s|>N_{k}}\left|\varphi^{(s)}(w) \varphi^{(s)}(a) e_{w}(a) / s !\right|
\end{gathered}
$$

But

$$
\begin{gathered}
\sum_{|s| \leq N_{k}}\left|\varphi^{(s)}(w)\left[\varphi^{(s)}(a)-p_{k}^{(s)}(a)\right]\right|(s !)^{-1} \\
\leq\left[\sum_{s} \frac{\left|\varphi^{(s)}(w)\right|^{2}}{s !} \cdot \sum_{s}\left|\left(T_{e_{a}}^{*}\left(\varphi-p_{k}\right), f_{s}\right)\right|^{2}\right]^{1 / 2}
\end{gathered}
$$

The last sum can be estimated by

$\left\|T_{e_{a}}^{*}\left(\varphi-p_{k}\right)\right\|^{2} \leq\left\|e_{a}\left(\varphi-p_{k}\right)\right\|^{2}$ and so using $(\alpha)$ and $(\beta)$ we have $A_{k} \leq \varepsilon$, for $k \geq k_{0}$.

It follows that

$$
\lim _{k} \sum_{s} \frac{\overline{\varphi^{(s)}(w)} p_{k}^{(s)}(a)}{s !}=\sum_{s} \frac{\overline{\varphi^{(s)}(w)} \varphi^{(s)}(a)}{s !}
$$

In virtue of $(\gamma)$ we have 


$$
\begin{aligned}
& \left(\Pi_{\bar{\varphi}}\left(\varphi e_{w}\right), e_{a}\right)=\left(\varphi e_{w}, \varphi e_{a}\right)=\lim _{k}\left(e_{w}, p_{k}^{*}(D)\left(\varphi e_{a}\right)\right) \\
= & \lim _{k}\left(e_{w}, \sum_{s} \frac{p_{k}^{*(s)}(D) e_{a} \cdot \varphi^{(s)}}{s !}\right)=\lim _{k} \sum_{s} \frac{\overline{\varphi^{(s)}(w)} p_{k}^{(s)}(a) e_{w}(a)}{s !} \\
= & \sum_{s} \frac{\overline{\varphi^{(s)}(w)} \varphi^{(s)}(a) e_{w}(a)}{s !}=\left(\sum_{s} \frac{\overline{\varphi^{(s)}(w)} \varphi^{(s)}(\cdot)}{s !} e_{w}, e_{a}\right)
\end{aligned}
$$

and this proves our claim.

Suppose that there exists a function $f \in D\left(T_{\varphi}\right)$ such that

$$
\left(f, e_{w}\right)+\left(\varphi f, \varphi e_{w}\right)=0, \quad w \in \mathrm{C}^{n} .
$$

By applying Proposition 1.4 of [6] it is enough to show that $f=0$.

Due to (3.1) we may rewrite (3.2) as

$$
\left(f, \sum_{s} \frac{\overline{\varphi^{(s)}}(w) \varphi^{(s)} e_{w}}{s !}\right)+f(w)=0 .
$$

Multiplying the above equality by $\overline{f(w)}$ we have

$$
\overline{f(w)}\left(f, \sum_{s} \frac{\overline{\varphi^{(s)}(w)} \varphi^{(s)} e_{w}}{s !}\right)+|f(w)|^{2}=0
$$

Consider the sequence of functions given by

$$
F_{N}(w):=\left(f, \sum_{s=0}^{N} \frac{\overline{\varphi^{(s)}}(w) \varphi^{(s)} e_{w}}{s !}\right) \bar{f}(w) .
$$

Observe that $F_{N}$ has $L^{1}(\mu)$ majorant.

This is clear in view of $(* *)$

$$
\begin{gathered}
\left|F_{N}(w)\right| \leq|f(w)|\|f\| \sum_{s=0}^{N} \frac{\left|\varphi^{(s)}(w)\right|\left\|\varphi^{(s)} e_{w}\right\|}{s !} \\
\leq|f(w)|\|f\| \sum_{s=0}^{\infty} \frac{\left|\varphi^{(s)}(w)\right|\left\|\varphi^{(s)} e_{w}\right\|}{s !}
\end{gathered}
$$

Envoking (3.3) and the Lebesque theorem we have 


$$
\begin{gathered}
-\|f\|^{2}=\int_{N} \lim _{N} F_{N}(w) d \mu=\lim _{N} \int F_{N}(w) d \mu \\
=\lim _{N} \sum_{s=0}^{N} \frac{1}{s !} \int \Pi_{\bar{\varphi}^{(s)}} f(w) \overline{\varphi^{(s)}(w) f(w)} d \mu \\
=\lim _{N} \sum_{s=0}^{N} \frac{1}{s !}\left(\Pi_{\bar{\varphi}^{(s)}} f, \bar{\varphi}^{(s)} f\right) \\
=\lim _{N} \sum_{s=0}^{N} \frac{1}{s !}\left\|\Pi_{\bar{\varphi}^{(s)}} f\right\|^{2} \geq 0
\end{gathered}
$$

where in the last equality we used $\left(^{*}\right.$ ) (and the relation $T_{\bar{\varphi}^{(s)}} \subset \Pi_{\bar{\varphi}^{(s)}}$ ). It follows that $f=0$ and this completes the proof.

The problem of computing $T_{\varphi}^{*}$ for not necessarily entire $\varphi$ is rather diffficult. In [7] we have found some classes of symbols for which this problem was solved. Below we shall find a class of $\varphi$ for which $T_{\varphi}^{*}=T_{\bar{\varphi}}$. Surely, this is a special family of functions. Nevertheless, it seems to deserve mentionning.

Proposition 3.2. Let $\varphi$ be a Borel function in $\mathrm{C}^{n}$, which satisfies the condition

$$
|\varphi(z)| \leq M \exp \left(\rho\|z\|^{2}\right), \quad 0<\rho<\frac{1}{8}
$$

Define the linear space

$$
D=\left\{h \in D\left(M_{\varphi}\right): P h \in D\left(M_{\varphi}\right)\right\} .
$$

Then $\left(M_{\varphi \mid D}\right)^{-}=M_{\varphi}$

Proof. First note that $D$ contains the space $R$ of all polynomials in $z_{1}, \overline{z_{1}}$, $z_{2}, \overline{z_{2}}, \cdots, z_{n}, \overline{z_{n}}$. We claim that

$$
\left(M_{|\varphi|^{2} \mid R}\right)^{-}=M_{|\varphi|^{2}} .
$$

Let $d \mu_{r}=\left(\frac{r}{\pi}\right)^{n} e^{-r\|z\|^{2}} d V, r>0$. It is well known that $R$ is dense in $L^{2}\left(\mu_{r}\right)$ (cf. [1]). Hence for any $h \in D\left(M_{|\varphi|^{2}}\right)$ there exist a sequence $p_{k} \in R$ such that

$$
\int\left|h-p_{k}\right|^{2} d \mu_{r} \rightarrow 0 \quad \text { as } \quad k \rightarrow \infty
$$

where $r=1-8 \rho$. But

$$
\int|\varphi|^{4}\left|\left(h-p_{k}\right)\right|^{2} d \mu \leq M r^{-n} \int\left|h-p_{k}\right|^{2} d \mu_{r},
$$

and so $(*)$ holds true. 
Now suppose that there is $k \in D\left(M_{\varphi}\right)$ for which $\left(k, M_{\varphi} k\right)$ is orthogonal to $\left(h, M_{\varphi} h\right)$, for every $h \in R$, i.e.

$$
\left(M_{\varphi} k, M_{\varphi} h\right)+(k, h)=0, \quad h \in R .
$$

It is obvious (by (+)) that $M_{\varphi} h \in D\left(M_{\varphi}^{*}\right)=D\left(M_{\varphi}\right)$ for $h \in R$.

Therefore (3.4) is equivalent to

$$
\left(k,\left(M_{|\varphi|^{2}}+I\right) h\right)=0, \quad h \in R .
$$

Since, in virtue of $(*), R$ is a core for $M_{|\varphi|^{2}}, k$ is orthogonal to $R\left(M_{|\varphi|^{2}}+I\right)=$ $L^{2}(\mu)$. This completes the proof.

Applying Proposition 3.2 we have.

THEOREM 3.3. If $\varphi$ satisfies the condition

$$
(++) \quad|\varphi(z)-\varphi(w)| \leq C e^{\|z-w\|^{2} / 4} \text {, then } T_{\varphi}^{*}=T_{\bar{\phi}}
$$

Proof. Let $A_{\varphi}$ be the operator in $L^{2}(\mu)$ given by the operator matrix (with respect to the decomposition $L^{2}(\mu)=B \oplus B^{\perp}$ ):

$$
\left(\begin{array}{cc}
T_{\varphi} & H_{\varphi}^{*} \\
H_{\varphi} & R_{\varphi}
\end{array}\right)
$$

where $D\left(A_{\varphi}\right):=D\left(T_{\varphi}\right) \oplus D\left(R_{\varphi}\right)$ and $D\left(R_{\varphi}\right)=D\left(M_{\varphi}\right) \cap B^{\perp}$, $R_{\varphi} u=(I-P) M_{\varphi} u$.

The above definition is correct because (++) implies that $H_{\varphi}$ and $H_{\bar{\varphi}}^{*}$ are bounded operators. (see [7]). Moreover, simple reasoning proves that boundedness of $H_{\varphi}$ and $H_{\bar{\varphi}}^{*}$ implies closedness of $T_{\varphi}$ and $R_{\varphi}$. The inclusions $D\left(A_{\varphi}\right) \supset D$ and $M_{\varphi} \supset A_{\varphi}$ are obvious.

Since $\varphi(z)$ satisfies the inequality $(++)$, one can easily check that

$$
|\varphi(z)| \leq \frac{3}{2} C \max \left\{1, r^{-1}\right\} e^{r\|z\|^{2}}
$$

for all $z \in \mathrm{C}^{n}$ and $r>0$ (see [3]). It follows that $\varphi$ must satisfy the above inequality (+). Hence in virtue of Proposition 3.2 we have

$$
M_{\varphi}=\left(M_{\varphi \mid D\left(A_{\varphi}\right)}\right)^{-}=\left(\begin{array}{cc}
T_{\varphi} & 0 \\
0 & R_{\varphi}
\end{array}\right)+\left(\begin{array}{cc}
0 & H_{\varphi}^{*} \\
H_{\varphi} & 0
\end{array}\right) .
$$

In the last equality we also used the relation $\overline{A+B}=\bar{A}+B$, which holds for any bounded operator $B$. It follows that

$$
D\left(T_{\varphi}^{*}\right) \oplus D\left(R_{\varphi}^{*}\right)=D\left(M_{\varphi}^{*}\right)=D\left(M_{\varphi}\right)=D\left(T_{\varphi}\right) \oplus D\left(R_{\varphi}\right)
$$


Therefore for any $f \in D\left(T_{\varphi}^{*}\right)$ there exist $f_{1} \in D\left(T_{\varphi}\right)$ such that $f=f_{1}$. Since $T_{\varphi}^{*} \supset T_{\bar{\varphi}}$ this completes the proof.

Note also (for the future use) the following simple result.

Proposition 3.4. If $T_{\varphi}^{*}=\bar{T}_{\bar{\varphi}}$, then

$$
\sigma\left(\bar{T}_{\varphi}\right) \subset \operatorname{clconv} \sigma\left(M_{\varphi}\right) \cup \operatorname{clconv} \sigma\left(M_{\bar{\varphi}}\right)
$$

where clconv $\sigma\left(M_{\varphi}\right)$ is the closed convex hull of $\sigma\left(M_{\varphi}\right)$.

Proof. By general theory we know that

$$
\sigma\left(\bar{T}_{\varphi}\right) \subset \operatorname{cl} W\left(\bar{T}_{\varphi}\right) \cup \operatorname{cl} W\left(T_{\varphi}^{*}\right) .
$$

For any $f \in D\left(T_{\varphi}^{*}\right)$ there exist a sequence $\left\{f_{k}\right\} \subset D\left(T_{\varphi}\right)$ s.t.

$$
\left(T_{\varphi}^{*} f, f\right)=\lim _{k} \int \bar{\varphi}\left|f_{k}\right|^{2} d \mu \in \operatorname{clconv} \sigma\left(M_{\bar{\varphi}}\right) .
$$

This imples the desired inclusion.

In particular, Proposition 3.4 can be applied to any $\varphi$ satisfying the condition $(++)$ of Th. 3.3 .

\section{Toeplitz Operators As Generators Of Contraction Semigroups}

It is of interest to know when Toeplitz operators generate contraction semigroups. Unfortunately, there are no nontrivial estimations of the resolvent of Toeplitz operators and the above question is far from being answered.

We have found only a few classes of symbols $\varphi$ which induce $T_{\varphi}$ generating a contraction semigroup. First note the following corollary of Proposition 3.4.

Proposition 4.1. If $D\left(T_{\varphi}^{*}\right)=D\left(\bar{T}_{\varphi}\right)$ and $\operatorname{Re} \varphi \leq 0$, then $\bar{T}_{\varphi}$ generates a contraction semigroup.

Proof. In virtue of Proposition 3.4 we know that $\sigma\left(\bar{T}_{\varphi}\right) \subset \mathrm{C}^{-}=$ $\{\lambda \in \mathrm{C}, \operatorname{Re} \lambda \leq 0\}$. Since $W\left(\bar{T}_{\varphi}\right) \subset \mathrm{C}^{-}$, for any $\lambda \notin \mathrm{C}^{-}$we have

$$
\left\|\left(\lambda-T_{\varphi}\right)^{-1}\right\| \leq \frac{1}{\operatorname{dist}\left(\lambda, W\left(\bar{T}_{\varphi}\right)\right)} \leq \frac{1}{\operatorname{Re} \lambda}
$$

and this estimate completes the proof.

The last result is not easy to check in practice because of the assumption $D\left(T_{\varphi}^{*}\right)=D\left(\bar{T}_{\varphi}\right)$. Therefore we shall give below another criterion for $\bar{T}_{\varphi}$ to generate a contraction semigroup. 
Proposition 4.2. Suppose we are given a function $\varphi$ such that $\operatorname{Re}\left(T_{\varphi} f, f\right) \leq 0, f \in D\left(T_{\varphi}\right)$. If $T_{\varphi}$ maps the space $P_{N}$ of polynomials of degree less or equal to $N$ into itself, then $\bar{T}_{\varphi}$ generates a contraction semigroup.

Proof. Since $T_{\varphi}$ is dissipative (by our assumption) and $P_{N}$ are finite dimensional we may apply Proposition 3.5 from [4] and obtain the desired result.

In order to illustrate the last Proposition we have the following example.

Example 4.3. Let $\varphi_{0}(z, \bar{z})=\sum_{s} q_{s}(z) \bar{z}^{s}$ (a finite sum), where $q_{s}\left(s \in \mathbf{Z}_{+}^{n}\right)$ are polynomials such that $\operatorname{deg} q_{s} \leq|s|=s_{1}+\cdots+s_{n}$. Suppose that $\operatorname{Re} \varphi_{0}(z, \bar{z}) \leq 0, \quad z \in \mathrm{C}^{n}$. It is easy to check that $T_{\varphi_{0}}: P_{N} \rightarrow P_{N}$. Hence $\bar{T}_{\varphi_{0}}$ generates a contraction semigroup.

We conclude this section with yet another approach to the problem of Toeplitz operators as generators of contraction semigroups. This approach relies on the construction of such generators due to Lions [9]. Suppose we are given a Borel function $\varphi$ on $\mathrm{C}^{n}$ such that $\operatorname{Re} \varphi \geq \alpha>0$. Consider the subspace $V \subset B$ defined as

$$
V=\left\{f \text { - entire, } \int \operatorname{Re} \varphi(z)|f(z)|^{2} d \mu(z)<+\infty\right\} .
$$

Assume that $V$ is dense in $B$. Introduce in $V$ a Hilbert space structure by the norm

$$
|f|_{1}^{2}:=\int \operatorname{Re} \varphi(z)|f(z)|^{2} d \mu(z) .
$$

Suppose that $\varphi$ satisfies additionally the following condition

$$
|\operatorname{Im} \varphi(z)| \leq C \operatorname{Re} \varphi(z), \quad \text { a.e. in } \mathrm{C}^{n} .
$$

Let $a(u, v):=-\int \varphi(z) u(z) \bar{v}(z) d \mu$, be the sesquilinear form on $V$. It is clear that $a(.,$.$) is continuous on V\left(\right.$ with $|\cdot|_{1}$ norm ). Define

$$
D\left(A_{\varphi}\right)=\{u \in V \text {, the functional } a(u, \cdot) \text { is continuous in the }\|\cdot\| \text { norm }\} .
$$

Since $V$ is dense in $B$ for any $u \in D\left(A_{\varphi}\right)$, there exists exactly one $A_{\varphi} u \in B$ such that

$$
\left(A_{\varphi} u, v\right)=a(u, v)
$$

Moreover, we have

$$
\operatorname{Re} a(u, u)+\alpha\|u\|^{2} \leq 0
$$


(because $\operatorname{Re} \varphi \geq \alpha$ ). Hence by a result of Lions we know that $A_{\varphi}$ is a generator of a contraction semigroup [9, Th 1.2, p. 116].

Proposition 4.4. Let $\varphi$ be a Borel-measurable function satisfying the assumptions $\operatorname{Re} \varphi \geq \alpha>0$ and (c). Assume that $E \subset V$ ( $E$ as above).

Then $A_{\varphi} \subset-\Pi_{\varphi}$.

Proof. Take $u \in D\left(A_{\varphi}\right)$. Since $e_{w} \in V$, we have $a\left(u, e_{w}\right)=-\int \varphi \bar{e}_{w} d \mu=$ $\left(A_{\varphi} u, e_{w}\right)=\left(A_{\varphi} u\right)(w)$. But $w \in \mathrm{C}^{n}$ is arbitrary, therefore $u \in D\left(\Pi_{\varphi}\right)$ and

$$
-\Pi_{\varphi} u(w)=\left(A_{\varphi} u\right)(w) .
$$

\section{A Result On Fischer Pairs}

In [11] Shapiro introduced the notion of Fischer pairs. Let $\mathscr{E}$ be the space of all entire functions in $\mathrm{C}^{n}$.

Definition 5.1. The pair $p, q$ of polynomials forms a Fischer pair if the $\operatorname{map} f \rightarrow q(D)(p f)$ is a bijection of $\mathscr{E}$.

We restrict the above notion to the context of Hilbert space $B$ i.e. $p, q$ is a Fischer pair if the map $f \rightarrow T_{q}^{*} T_{p} f$ is a bijection carrying its domain onto $B$. Shapiro proved in [11] that for any homogeneous polynomial $p(\neq 0)$ and $c \in \mathrm{C}$ the pair $p, p+c$ forms a Fischer pair. We tried to extend this result for not necessarily homogeneous $p$ in the space $B$. It turned out that perturbation theory of Hilbert space operators provides a suitable tool for such extension. We found a simple abstract version of a Fischer pair $p, p+c$.

Before stating this version recall that for a closed operator $T$ in a Hilbert space $H$ such that $\operatorname{ker} T=\{0\}$, the minimum modulus of $T$ is given by $\gamma=\gamma(T)=\inf \{\|T x\|,\|x\|=1\}$. If $T$ is a Fredholm operator, then ind $T=\operatorname{dim} \operatorname{ker} T-\operatorname{dim} \operatorname{ker} T^{*}$.

THeOREM 5.2. Let $T$ be a closed operator in H. Suppose that

$$
\|T x\| \geq(1+\epsilon)\|x\|, \quad \epsilon>0 .
$$

Then the operator $S=T^{*} T-T$ defined on $D\left(T^{*} T\right)$ is a bijection from $D(S)$ onto $H$.

Proof. First note that

$$
\|T x\| \leq \frac{1}{2}\left[\|x\|+\left\|T^{*} T x\right\|\right], \quad x \in D\left(T^{*} T\right) .
$$

Indeed, we have

$$
\|T x\|=\left(T^{*} T x, x\right)^{\frac{1}{2}} \leq\left[\left\|T^{*} T x\right\| \cdot\|x\|\right]^{\frac{1}{2}} \leq \frac{1}{2}\left(\left\|T^{*} T x\right\|+\|x\|\right) .
$$


Since $\left\|T^{*} T x\right\|\|x\| \geq\|T x\|^{2}$, the condition $(*)$ implies that $\gamma\left(T^{*} T\right)>1$. Moreover, the condition (*) also forces that $\operatorname{ker} S=\{0\}$.

Now, $T^{*} T$ is invertible (hence Fredholm) and so applying Theorem 5.2 from [8, p. 236] with $a=b=\frac{1}{2}$ (use (5.1)) we know that $S$ is Fredholm and ind $S=$ ind $T^{*} T=0$. Therefore $R(S)=H$ and the proof is complete.

COROLlary 5.3. Let $p$ be a polynomial such that

$$
\|p f\| \geq(1+\epsilon)\|f\|, \quad \epsilon>0, f \in D\left(T_{p}\right) .
$$

Then the $p, p+c$ is a Fischer pair in $B$ for $|c|<1+\varepsilon$.

Proof. Let $r=p / c$ (we may assume that $c \neq 0$ ).

Since $T_{p}$ is closed in B and satisfies (5.2) it follows that

$$
\|r f\| \geq \frac{1+\varepsilon}{|c|}\|f\|=\left(1+\varepsilon^{\prime}\right)\|f\|, \quad \varepsilon^{\prime}=\frac{1+\varepsilon-|c|}{|c|} .
$$

The result follows easily.

Remark 5.4. Note that we could extend Corollary 5.3 to a general entire $\varphi$, provided $T_{\varphi}$ is densely defined and

$$
\|\varphi h\| \geq(1+\epsilon)\|h\|, \quad h \in D\left(T_{\varphi}\right) .
$$

Remark 5.5. Corollary 5.3 has still another extension. Consider namely a densely defined operator $H$ in $H$ which satisfies the so called "generalized canonical commutation relation" i.e. there exists a dense linear subspace $M \subset D(S) \cap D\left(S^{*}\right)$ such that

$$
S M \subset M, S^{*} M \subset M \text { and } S^{*} S h-S S^{*} h=F^{2} h, \quad h \in M,
$$

where $F$ is symmetric and commutes with $S$ on $M$. Then for any polynomial $p(\neq 0)$ there exists a constant $\delta>0$ (which depends on $p$ ) such that

$$
\|p(S) h\| \geq \delta\|h\|, \quad h \in M,(\text { see }[5]) .
$$

It may happen (as some examples show) that $\delta>1$. Then one can apply Theorem 5.2 to the operator $T=\left(\left.p(S)\right|_{M}\right)^{-}$. In fact (5.3) implies that $T$ satisfies the condition $(*)$ of Theorem 5.2.

\section{Concluding Remarks And Problems}

We studied in the paper (and in our previous ones) some properties of Toeplitz operators in $B$. However there are still many unanswered questions one can ask about them.

One of such questions we may ask, is to find a class of symbols $\varphi$, which induce nonnegative $T_{\varphi}$. For example let 


$$
\varphi_{0}\left(z_{1}, z_{2}\right)=\left|z_{1} z_{2}\right|^{2}+\operatorname{Re}\left(z_{1} \bar{z}_{2}\right) .
$$

Then $T_{\varphi_{0}}$ is non-negative. Note that $\varphi_{0}$ is far from being positive in $\mathrm{C}^{2}$.

The next natural question is: when the square root of a Toeplitz operator remains Toeplitz?

Since $A^{1 / 2} h=\frac{1}{\pi} \int_{0}^{\infty} \lambda^{-\frac{1}{2}}(A+\lambda)^{-1} A h d \lambda$, the above question is strongly related to the problem (posed already by Berger and Coburn in [2]), when the product of Toeplitz operators is again Toeplitz.

This research was supported by the KBN Grant 220349203

\section{REFERENCES}

1. F.A. Berezin, Wick and anti-Wick symbols of operators (in Russian), Mat. Sb. 86 No. 4 (1971), 578-610.

2. C.A. Berger and L.A. Coburn, Heat flow and Berezin-Toeplitz estimates, Amer. J. Math. 116 (1994), 563-590.

3. D.Cichoń and J.Stochel, On Toeplitz operators in Segal-Bargmann spaces, Univ. Iagel. Acta Math. (to appear).

4. S.N. Ethier and T.G. Kurtz, Markov Processes. Characterization and convergence, Wiley Series in Probability and Mathematical Statistics, New York, John Wiley \& Sons (1986).

5. J. Janas, Note on generalized commutation relations, Proceed. of the 6-th Symp. "Classical Analysis" ed. T. Mazur, World Scientific (1991), 46-52.

6. J. Janas, Unbounded Toeplitz operators in the Bargmann-Segal space, Studia Math. 99 (1991), 87-99.

7. J. Janas and J. Stochel, Unbounded Toeplitz operators in the Segal-Bargmann space II, J. Funct. Anal. 126 (1994), 418-447.

8. T. Kato, Perturbation Theory for Linear Operators, Springer Verlag, Second Edition (1976).

9. J.L. Lions, Controle optimale de systémes gouverné par des équations aux dérivées partielles, Dunod, Paris (1968).

10. D.J. Newman and H.S. Shapiro, Fischer spaces of entire functions, Proc. Sympos. Pure Math. XI "Entire functions and related parts of analysis" J. Korevar (Ed.) (1968), 360369.

11. H.S. Shapiro, An algebraic theorem of E. Fischer and the holomorphic Goursat problem, Bull. London Math. Soc. 21 (1989), 51-537.

INSTITUTE OF MATHEMATICS PAN

31-027 CRACOW

UL. ŚW. TOMASZA 30

POLAND

Email: najanas@cyf-kr.edu.pl 